ENCYCLOPEDEE Encyclopédie berbère

BERBERE

$11 \mid 1992$

11 | Bracelets - Caprarienses

\title{
Bubalus antiquus
}

Voir Art rupestre, Encyclopédie berbère, VI, p. 918-939.

G. Camps

\section{OpenEdition}

Journals

Édition électronique

URL : http://journals.openedition.org/encyclopedieberbere/1875

DOI : $10.4000 /$ encyclopedieberbere. 1875

ISSN : 2262-7197

Éditeur

Peeters Publishers

Édition imprimée

Date de publication : 1 septembre 1992

Pagination : 1642-1647

ISBN : 2-85744-581-4

ISSN : 1015-7344

Référence électronique

G. Camps, "Bubalus antiquus », Encyclopédie berbère [En ligne], 11 | 1992, document B111, mis en ligne le 01 mars 2013, consulté le 25 septembre 2020. URL : http://journals.openedition.org/ encyclopedieberbere/1875; DOI : https://doi.org/10.4000/encyclopedieberbere.1875

Ce document a été généré automatiquement le 25 septembre 2020.

(c) Tous droits réservés 


\section{Bubalus antiquus}

Voir Art rupestre, Encyclopédie berbère, VI, p. 918-939.

\section{G. Camps}

L'Afrique du Nord et le Sahara possèdent de nombreuses gravures rupestres représentant un buffle, espèce fossile connue depuis 1851 par la découverte d'un crâne fragmentaire dans les alluvions du Bou Sellam, près de Sétif (Algérie). Après avoir étudié ces restes, Duvernoy créait l'espèce Buffelus antiquus, appellation à laquelle Pomel, qui disposa de fossiles plus nombreux, préféra celle de Bubalus antiquus. L'étude de Pomel reposait sur un squelette presque complet, aux os remarquablement conservés, découvert en 1872 près de Djelfa; cette découverte avait été précédée de celle d'un autre crâne sur les bords du Rhummel, à Aïn Smara en 1859. Dans ces célèbres Monographies de la Carte de Géologie de l'Algérie (1893), Pomel, en s'appuyant à la fois sur les données ostéométriques et les figurations rupestres décrivait ainsi le Bubalus antiquus: "Une forte tête portée basse et oblique, un garrot très élevé en s'arrondissant, un rein large, une croupe plus ou moins anguleuse et très avalée, une queue courte, grêle, poilue au bout, pendante ou rejetée flexueusement sur la fesse, des membres très robustes et épais avec charpente puissante, donnent à cet animal une physionomie caractéristique et constituent les points les plus remarquables de son signalement " (p. 89-90). Il ajoutait que les extrémités des énormes cornes, qui décrivent un arc de cercle régulier et continu sont distantes de $2,40 \mathrm{~m}$, que le front, fortement bombé en tous sens et très développé en largeur, s'étend presque des orbites jusqu'à la crête occipitale. La position des orbites, tout près du départ des cornes, est caractéristique des buffles. Les artistes néolithiques, qui ont si souvent représenté cet animal n'ont pas omis ce détail, alors qu'ils figuraient plus bas, sur les figures du grand style naturaliste, l'œil du Bos Primigenius et de ses descendants domestiques. Mais c'est surtout par son cornage que le Bubalus antiquus se distingue des autres bovines. L'ampleur de ses cornes laisse entendre que cet animal vivait dans des espaces dégagés et peu arborés. Pomel, en s'appuyant sur les données ostéométriques, proposait les dimensions suivantes pour l'animal vivant: longueur de l'extrémité du mufle à la naissance de la queue : $3 \mathrm{~m}$ - hauteur du garrot : 1,85 $\mathrm{m}$ - hauteur de l'arrière-train : $1,70 \mathrm{~m}$. 
2 Les nombreuses découvertes de fossiles se rapportant à cette espèce n'ont pas modifié la description faite depuis un siècle. Nous signalerons toutefois que les mensurations faites sur l'atlas de l'espèce pléistocène (gisement des Allobroges à Alger) laissent entendre qu'elle était encore plus grande et plus robuste que la variété connue par le squelette de Djelfa; de plus, les figurations de Bubalus antiquus du Sahara Central donnent une silhouette moins lourde à cet animal, sans qu'on puisse dire s'il s'agit d'une variété différente ou d'un trait stylistique. Connu dès le Pléistocène inférieur (Aïn Hanech), le Bubalus antiquus a été trouvé dans plusieurs gisements du Pléistocène moyen (Aboukir, Ternifine, Tihodaïne...) et plus fréquemment encore, dans ceux du Pléistocène supérieur (El Aliya, Allobroges, Ali Bacha, Grottes du littoral d'Alger) et dans une quinzaine au moins de sites néolithiques. Moins fréquent au Sahara, le buffle antique y est aussi moins représenté dans l'art rupestre. Des éléments de son squelette ont été recueillis dans les zones d'épandage des oueds alors puissants qui dévalaient des massifs centraux (Tihodaïne, Amekni, Ouan Mouhouggiag) ainsi que dans le Désert égyptien occidental (Bir Terfawi). Le Bubalus antiquus fut donc une espèce présente dans tout le Nord du Continent africain; ses ossements et ses représentations rupestres peuvent être rencontrés sur un espace immense qui va de la Saguiat el-Hamra, à l'ouest au Nil, soudanais, à l'est, et du littoral méditerranéen jusqu'au sud du Sahara.

Deux buffles (Bubalus antiquus) gravés de la station de Kef el Akhal ; ces deux figurations presque identiques appartiennent au grand style naturaliste. Relevé $\mathrm{H}$. Lhote.

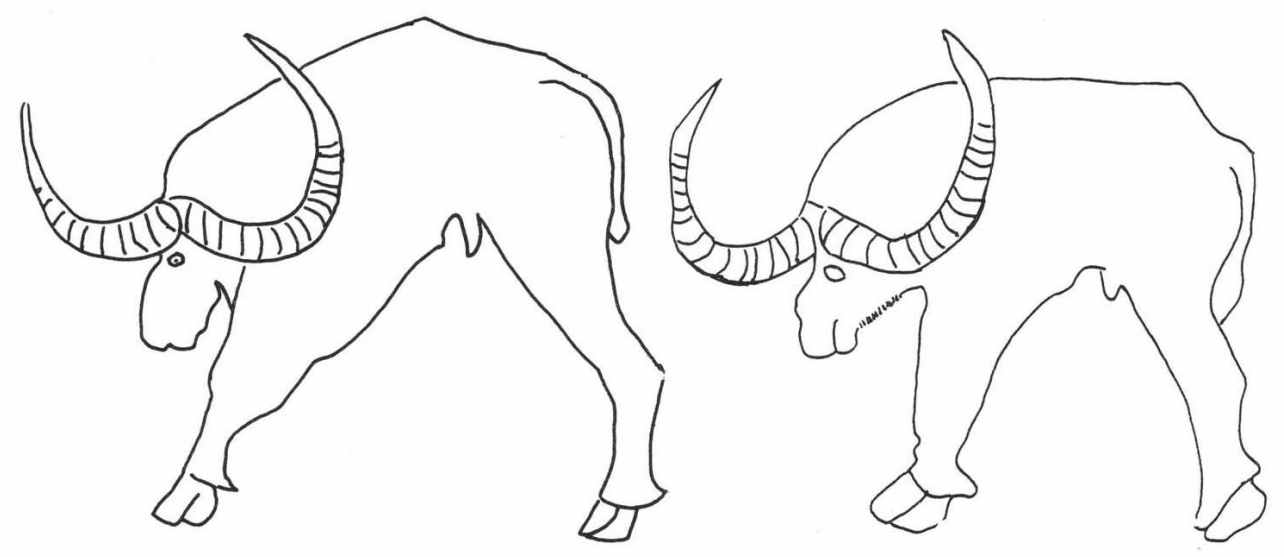

Cette espèce reçut des noms successifs qui révèlent les hésitations ou les doutes des paléontologues quant à son origine et ses affinités. Au Buffelus antiquus de Duvernoy, A. Pomel, nous l'avons vu, préféra la dénomination Bubalus antiquus qui fut d'un usage exclusif pendant la première moitié de ce siècle, puis D.M.A. Bate (1949) proposa de le nommer Homoïoceras antiquus à partir d'ossements recueillis en Afrique de l'est et du sud. Cette nouvelle appellation n'avait pas encore éliminé la précédente que le buffle antique de l'Afrique du Nord était assimilé à l'espèce Pelorovis ou Giant Buffalo ; enfin dans une communication au Congrès sur «L'Arte e l'ambiante del Sahara preistorico » (Milan, octobre 1990), A. Gauthier se déclarait « de plus en plus convaincu que ce bovidé appartient à la lignée des buffles actuels africains (Syncerus afer) dont il représentait une très grande forme adaptée à des biotopes très ouverts. "

Devant une telle avalanche taxinomique, nous préférons conserver l'ancienne appellation spécifique de Bubalus antiquus qui a le mérite d'avoir été utilisée depuis très 
longtemps et qui est communément employée, avec les inconvénients que nous verrons infra, dans l'étude de l'art rupestre africain.

Représentation stylisé de Bubalus antiquus de Fedj Naam (style de Tazina). Relevé H. Lhote.

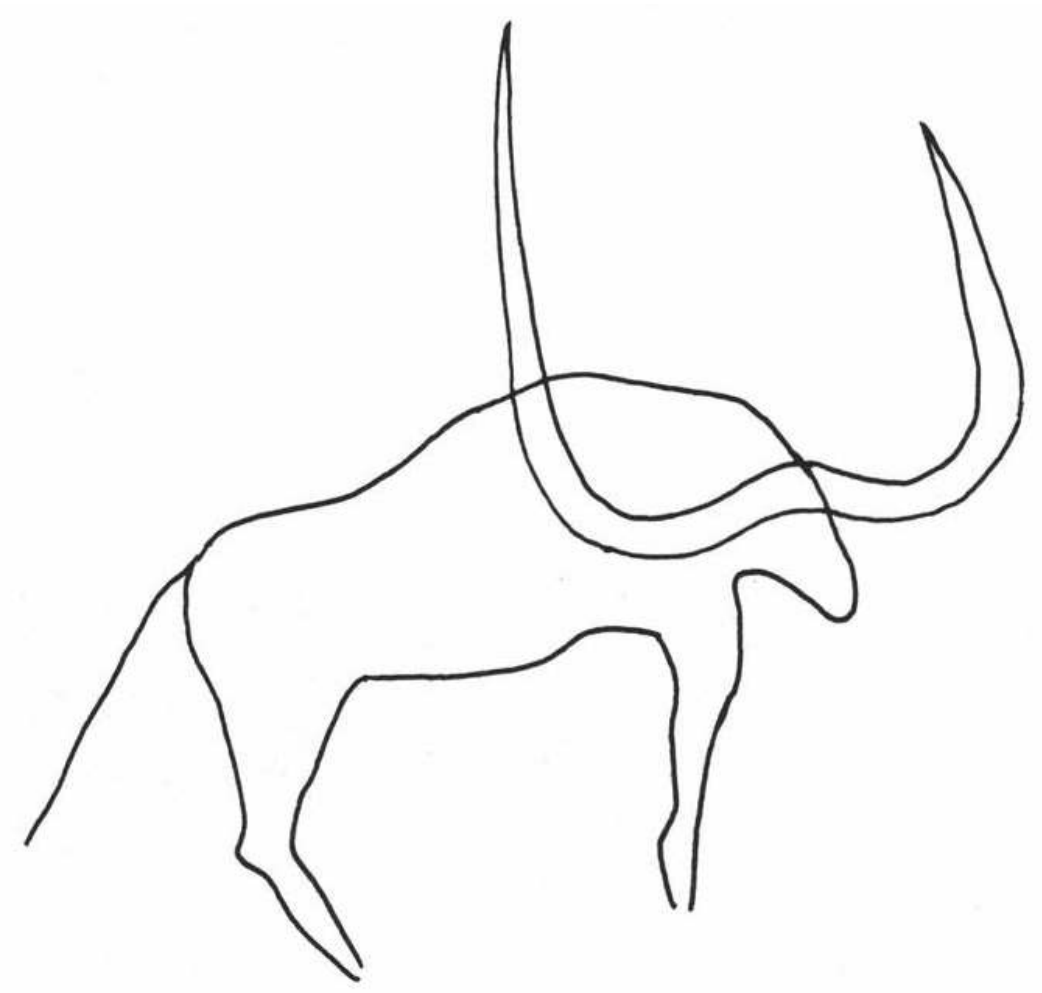

5 Le Bubalus antiquus occupe, en effet, une place de choix dans l'art de l'Atlas et dans celui du Sahara. Bien que tout dénombrement soit sujet à discussion, il ne me paraît pas inutile de tenter de faire le décompte, à la suite de M. Hachid (1978), des représentations de ce puissant animal. Des figures du Bubalus antiquus existent dans toute cette vaste zone quasi ininterrompue de gravures rupestres qui s'étend de l'Atlantique jusqu'au confins de la Tunisie: on connaît 2 représentations de Bubalus antiquus dans la Saguiat el-Hamra, 4 dans le Sud Marocain, 5 dans les Monts des Ksours, région d'Aïn Sefra, 57 dans la région d'El Bayadh, 5 dans le Djebel Amour, région d'Aflou, 25 dans les Monts des Ouled Naill, au sud de Djelfa. Au nord de cette zone, on connaît encore 3 représentations dans la région de Tiaret et autant dans celle de Constantine, enfin à l'est les dernières figurations sont les 7 reconnues dans les Nemencha et la région de Tébessa. Ce dénombrement imparfait qui comptabilise 136 représentations de Bubalus antiquus est cependant plus proche de la vérité que celui tenté pour le Sahara où on ne connaît qu'une quarantaine de figurations dont 11 pour la seule vallée de l'Oued Djerat. 
Troupeau de Bubalus antiquus de Wadi in-Elobu (Messak Settafet). Photo M. Van Albada.

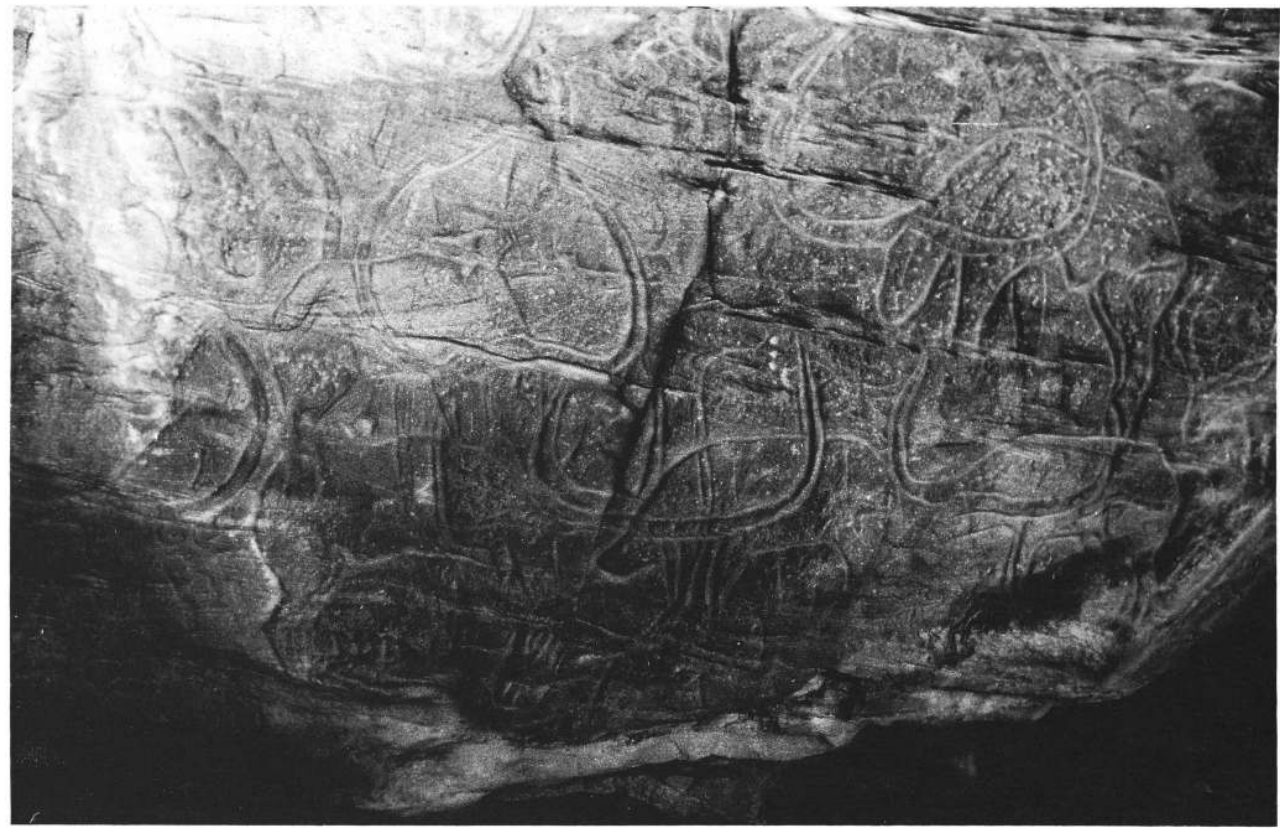

6 Au Maghreb, même si l'animal n'est pas toujours représenté avec le maximum de réalisme qui caractérise le style ancien, les attitudes et traits caractéristiques sont constants. L'animal est toujours représenté de profil absolu, seules les deux pattes du côté du spectateur sont figurées (l'unique exception est constituée par la scène de combat de l'oued Azouania où une troisième patte est figurée sur l'un des buffles) alors que les cornes sont représentées de face décrivant un grand arc de cercle; quand l'œil est figuré, il est toujours, comme dans la nature, très proche de la base des cornes; il arrive parfois que par convention, les deux yeux soient placés côte à côte alors que la tête reste de profil. Dans les gravures de style naturaliste, les détails ne font pas défaut qui accentuent le réalisme de ces représentations : indication du sexe, du fanon, de la barbiche, de l'ergot à l'arrière et au-dessus des sabots, des cannelures de l'étui corné, de la queue qui fouette la croupe ; aussi des figures comme celles du Kel el-Akhal, de la Gada el-Kharrouba, de Gouiret bent Selloul ou de Aïn Sfissifa et de bien d'autres stations méritent d'être comptées parmi les chefs-d'œuvre de l'art rupestre nordafricain. Il faut leur ajouter les très belles scènes de combat entre deux buffles mâales, sujet traité à El Richa, à l'Oued Azouania, à Khrelouet Sidi Cheikh. Ces gravures sont parmi les plus grandes. Dans les décomptes réalisés par M. Hachid, à partir de 64 gravures permettant ces mesures, un peu plus de $15 \%$ sont à la grandeur naturelle, $40 \%$ aux $2 / 3$ de la grandeur naturelle et $45 \%$ au $1 / 3$.

7 Bien représenté dans le style naturaliste le plus ancien qui a donné ces œuvres de grande classe, le buffle antique continue à être représenté dans toutes les phases qui précèdent l'apparition du cheval domestique. On le reconnaît stylisé dans le style de Tazina et du Naturalisme "décadent ». Il est, au Sahara, contemporain des peintres bovidiens qui l'ont représenté en observant d'autres règles; mais même aux époques antérieures, les artistes sahariens représentèrent le Bubalus antiquus d'une manière différente de celle du Maghreb. Ainsi aussi bien à l'oued Djerat qu'au Fezzan les buffles ont presque toujours leur quatre pattes visibles et plus élancées, les corps sont plus légers, parfois même levrettes. 
Présent dès le début du Quaternaire, figuré dans toutes les phases de l'art rupestre néolithique, le Bubalus antiquus ne peut, malgré la qualité de certaines représentations du style naturaliste, servir à désigner cette époque de l'art préhistorique nord-africain et saharien. On a déjà développé les multiples arguments qui font rejeter l'expression style bubalin ou " période » bubaline (Encyclopédie berbère, VI, p. 920-922).

Comme pour toutes les œuvres d'art préhistorique, il est difficile de trouver un sens à la représentation de ce puissant animal qui devait impressionner autant que l'éléphant qui est encore plus souvent que lui représenté dans l'art rupestre nord-africain. Il est sûr que ces belles gravures avaient un sens symbolique et que, par exemple, une scène de la qualité du combat des buffles d'El Richa (cf. Encyclopédie berbère, VI, p. 918) dépassait la simple anecdote. Il n'existe aucune scène figurant explicitement une chasse au buffle bien que les spirales qui les accompagnent parfois aient été interprétées comme des lassos et que des hommes armés de hache aient pu être considérés comme des chasseurs. L'association de l'homme et du buffle est d'ailleurs peu fréquente, on ne dénombre qu'une douzaine de cas; l'homme ou les hommes représentés "touchent " l'animal sans qu'il soit possible de trouver dans cette scène la moindre vénération ou tentative d'apprivoisement. De telles relations entre l'homme et l'animal apparaissent dans d'autres scènes où figurent d'autres gosses espèces. De véritables orants (?), ou du moins des personnages ayant les bras levés, accompagnent le Bubalus antiquus dans plusieurs scènes: Kef Mektouba, R'cheg Dirhem, Kel el Akhal... mais aucune de ces scènes ne peut être mise en parallèle avec celles qui associent les béliers à sphéroïdes* aux orants.

Combat de Bubalus antiquus à El-Richa (Sud Oranais).

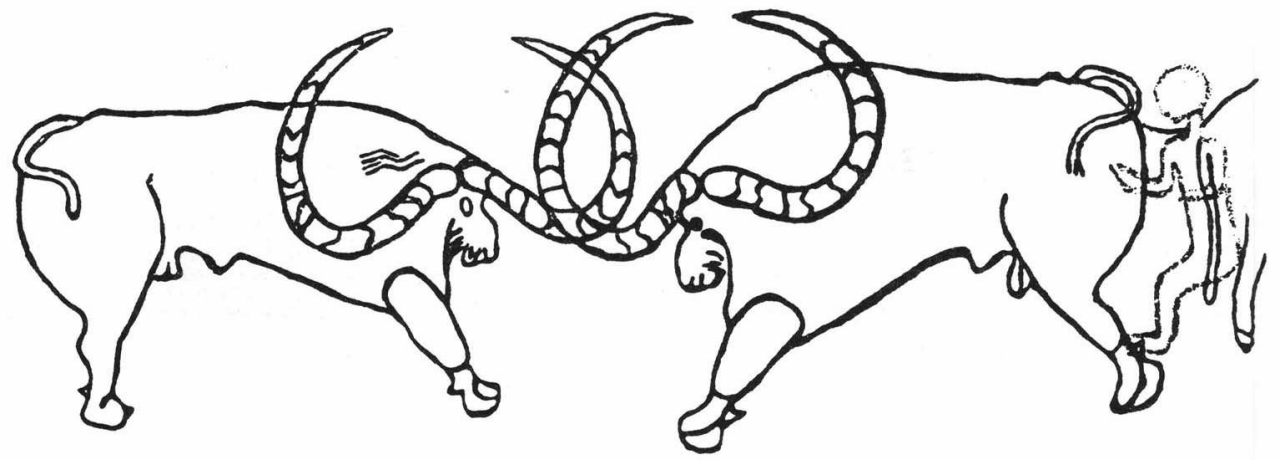

\section{BIBLIOGRAPHIE}

Voir Art rupestre, Encyclopédie berbère, VI, p. 918-939.

Duvernoy, Note sur une espèce de buffle antique, C.R. Acad. des Sc., Paris, décembre 1851.

Pomel A., Carte géologique de l'Algérie. Monographies. Bubalus antiquus, Alger, Fontana, 1893.

Bate D.-M., « A new african fossil long-horned buffalo », Ann. Mag. nat. history, 17, 1949, p. 396-398. 
Hachid M., Le Bubalus antiquus dans l'Art rupestre nord-africain et saharien, Université de Provence, Mémoire de Maîtrise, 1978.

INDEX

Mots-clés : Art rupestre, Tunisie, Zoologie 